\title{
The impact of tricuspid annular geometry on outcome after percutaneous edge-to-edge repair for severe tricuspid regurgitation
}

\author{
Sylvia Otto, Marija Velichkov, Ali Hamadanchi, \\ P. Christian Schulze, Sven Moebius-Winkler
}

Department of Internal Medicine I, Division of Cardiology, Angiology, and Intensive Medical Care, University Hospital Jena, Friedrich-Schiller-University Jena, Germany

\begin{abstract}
Background: Percutaneous tricuspid repair using the edge-to-edge technique is a novel treatment option. More data are needed to better understand which aspects predict a favorable outcome

Methods: Twenty high-risk patients (78.6 \pm 8.3 years, EuroScore II 9.1 $\pm 7.7 \%$, STS score $8.8 \pm 4.3$ ) with severe symptomatic tricuspid regurgitation (TR) were treated with the MitraClip ${ }^{\circledR}$ system. All patients underwent standardized pre-, peri-, and post-procedural evaluation. Acute success was defined as successful edge-to-edge repair with TR reduction of $\geq 1$ grade and survival until hospital discharge. Results: Fifteen (75\%) patients showed acute success until discharge and $12(60 \%)$ at 30-day followup. In $5(25 \%)$ patients repair failed due to either unsuccessful clip implantation $(n=2)$, single leaflet device attachment $(n=1)$, TR reduction $<1$ grade $(n=1)$, or in-hospital death $(n=1)$. Comparing patients with successful procedure versus those with failed repair revealed similar comorbidities but more severe right heart failure, lower left ventricular ejection fraction, worse renal function, and higher diuretic equivalent doses in the failed repair group. No differences in conventional echocardiographic parameters for TR severity but more dilated tricuspid annulus geometry (tricuspid valve annulus, coaptation depth, tenting area) in the failed repair group were observed. The success rate of non-central/ Inon-anteroseptal jet location was only $25 \%$.

Conclusions: Tricuspid annulus geometry assessment may be of crucial importance and seems to impact procedural outcomes in patients undergoing edge-to-edge tricuspid valve repair. Further investigations including advanced imaging are needed to better understand and treat this complex valve disease. (Cardiol J 2021; 28, 4: 579-588)
\end{abstract}

Key words: tricuspid regurgitation, percutaneous repair, transcatheter treatment, edge-to-edge technique, echocardiography

\section{Introduction}

Current guidelines reserve surgical treatment of severe tricuspid regurgitation (TR) almost exclusively to combined left-heart procedures, whereas indications for stand-alone surgery for severe TR are only vaguely described [1-3]. The tricuspid valve (TV) is commonly referred to as "the forgotten valve" because data on single TV surgery are sparse, randomized trials are lacking, and there are no definite criteria guiding indication and timing of TV repair [4-6]. TV disease is mainly of secondary nature due to left-sided heart disease, pulmonary hypertension, atrial fibrillation, or lead-related problems in patients with cardiac implantable electronic devices [7-9]. TV apparatus

Address for correspondence: Sylvia Otto, MD, Klinik für Innere Medizin I, Universitätsklinikum Jena, Am Klinikum 1, 07747 Jena, Germany, tel: +49 3641-9 3245 60, fax: + 49 3641-9 3241 02, e-mail: sylvia.otto@med.uni-jena.de

Received: 2.11 .2020

Accepted: 25.02.2021

This article is available in open access under Creative Common Attribution-Non-Commercial-No Derivatives 4.0 International (CC BY-NC-ND 4.0) license, allowing to download articles and share them with others as long as they credit the authors and the publisher, but without permission to change them in any way or use them commercially. 
anatomy and pathogenic mechanisms of TR are complex and involve various degrees of right heart remodeling and tricuspid annulus dysfunction. Echocardiographic grading of TR severity has limitations and seems insufficient despite introduction of a 5 -stage grading scheme in 2017 with expansion of the 'severe' grade into three subcategories [10]. Recently, a more integrated approach for evaluation of TR disease has been proposed $[7,11]$.

Patients with severe TR often represent a high-risk surgical population, which relies at least partly on late referral and advanced heart failure (HF) after left-sided heart surgery [12]. The prognosis of functional tricuspid regurgitation (FTR) is poor [13-15]. Benfari et al. [13] recently reported a 5 -year survival of $34 \%$ and a 10 -year survival of only $14 \%$ for patients with severe FTR and $\mathrm{HF}$ with reduced ejection fraction (HFrEF). Importantly, the excess mortality in TR seems to be independent of pulmonary hypertension and other concomitant clinical parameters, as recent studies suggest [13-17]. Therefore, it can be speculated that TV repair at an earlier disease stage might be favorable before irreversible right ventricular dysfunction and dilatation manifests [16, 18]. Such lessons were learned for the treatment of mitral valve regurgitation the past years. Because FTR is not only a marker for severity of HF disease, but also a major contributor in pathophysiological processes, it represents a potential therapeutic target in patients with HFrEF. Conversely, inhospital mortality of patients undergoing TV repair or replacement is substantial with reported rates between $8 \%$ and $24 \%[3,12,19,20]$. Older age ( $\geq 60$ years) and TV replacement instead of repair can further double the risk for in-hospital death [3].

A novel treatment option for severe TR in patients with high surgical risk is a percutaneous tricuspid valve intervention, where the edge-to-edge-technique using the MitraClip ${ }^{\circledR}$ is most often used. A variety of transcatheter treatment options have been developed which simulate different surgical approaches like suture or ring annuloplasty (e.g. Trialign, TriCinch, and Cardioband or Millipede, respectively), coaptation enhancement (MitraClip, Forma, Pascal), valve replacement (e.g. NaviGate, TriSol, Cavi/BiCavi), or neochordae repair (e.g. Tricentro, TricValve) [21]. Most are still under investigation and are being tested in different phases of research. Recently published data from the TriValve registry showed significant clinical improvement and reduction of 1-year mortality after successful transcatheter tricuspid edge-to-edge repair [22].
However, appropriate patient selection for percutaneous TR repair is of crucial importance. Data on parameters and clinical constellations that translate into procedural success and favorable clinical outcome after transcatheter TV intervention (TTVI) are limited.

The aim of this observational, prospective study was to analyze the clinical, echocardiographic, and procedural characteristics in patients with successful vs. unsuccessful percutaneous tricuspid intervention using the edge-to-edge technique.

\section{Methods}

\section{Study design and patient population}

All patients undergoing transcatheter TV repair at our institution were prospectively included in our TV registry. Here, we report our first experiences with edge-to-edge repair using the MitraClip ${ }^{\circledR}$ between $12 / 2017$ and $07 / 2019$ after starting this program in high-risk patients. All patients underwent standardized pre-, peri-, and post-procedural evaluation including clinical, echocardiographic and invasive examination. All parameters were prospectively entered into a database. All patients were discussed with the heart team and declined for conventional TV surgery. The safety and feasibility of the procedure, reduction of TR-grade, and clinical outcomes were collected and analyzed at the day of discharge and 30-day follow-up. Successful intervention was defined as successful edge-to-edge repair using one or more MitraClips ${ }^{\circledR}$ with TR reduction of $\geq 1$ grade and survival until hospital discharge.

The study was approved by the local ethics committee of the University Hospital of Jena (identification number: 2019-1325-BO) and conducted according to the principles of the Declaration of Helsinki.

\section{Statistical analysis}

All parameters were archived in a custommade database. Statistical calculations were done with SPSS (version 26.0, IBM SPSS statistics). Normal distribution was tested with the Shapiro-Wilks test. Continuous variables are expressed as mean \pm standard deviation and analyzed with the unpaired Student t-test. Categorical variables are presented as counts (percentages) and analyzed with $\chi^{2}$-test or Fisher's exact test for small patient numbers. Statistical significance was assumed for p-values $<0.05$ (two-tailed). 


\section{Results}

\section{Baseline clinical characteristics}

A total of 20 high-risk patients $(78.6 \pm 8.3$ years old, EUROScore II $9.1 \pm 7.7 \%$, Society of Thoracic Surgeons [STS] score $8.8 \pm 4.3$ ) with severe symptomatic TR were treated with the edge-to-edge technique using the MitraClip ${ }^{\circledR}$ system. Baseline clinical characteristics are presented in Table 1. Most patients were in New York Heart Association (NYHA) functional status class III and showed chronic peripheral edema and pleural effusion before TTVI (Table 1). All but 1 patient had concomitant atrial fibrillation, and 18 (90\%) patients showed pulmonary hypertension with a mean pulmonary pressure of $31.3 \pm 7.5 \mathrm{mmHg}$. We found concomitant mitral regurgitation of grade $\geq 2$ in 11 (55\%) patients. Overall, liver function was not reduced, and patients were on moderate dosage of diuretics $(24.2 \pm 15.6 \mathrm{mg}$ torasemid equivalent dose). Cardiopulmonary exercise test (spiroergometry) could only be performed in $8(40 \%)$ patients due to advanced HF and the frailty of the patient population (exercise capacity $59.1 \pm 20.6 \mathrm{~W}, \mathrm{VO}_{2}$ anaerobic threshold $9.1 \pm 1.6$, $\mathrm{VO}_{2}$ peak $\left.11.6 \pm 2.9\right)$. Cardiac index was $\leq 1.8 \mathrm{~L} /$ $/ \mathrm{min} / \mathrm{m}^{2}$ in $4(20 \%)$ patients. Concomitant percutaneous repair of the mitral valve, using the edgeto-edge technique as well, was done in $2(10 \%)$ patients before TTVI (Table 1).

\section{Tricuspid regurgitation:}

\section{Etiology and echocardiographic data}

Left ventricular (LV) systolic function was mostly normal or mildly reduced (ejection fraction [EF] $54.3 \pm 14.6 \%)$ and only $4(20 \%)$ patients had a LVEF below $40 \%$ (Table 2). Conversely, 11 (55\%) patients had a reduced right ventricle (RV) function with a RV fractional area change $<35 \%$ (Table 2). Our patient population showed a mean TR grade of $3.9 \pm 0.8$ (median 4.0) using the new 5 -stage grading scheme, with $13(65 \%)$ patients suffering from massive or torrential TR (Table 2, Fig. 1A, B) [10]. The effective regurgitation orifice area (EROA), TR volume, and TR vena contracta are outlined in Table 2. The etiology of TR was judged as functional in $14(70 \%)$ patients, structural in $5(25 \%)$ patients, and mixed in $1(5 \%)$ patient. Of note, chronic pulmonary disease was only noted in $3(15 \%)$ patients, and concomitant implantable cardioverter-defibrillator or pacemaker leads were detected in $3(15 \%)$ patients (Table 1$)$. The calculated mean TRuE risk score (https://thetruerisk. com [23]) of $7.1 \pm 2.3$ (median 6.0) in our sample size represents a high risk $\left(75^{\text {th }}\right.$ percentile) for 5 -year mortality and therefore a patient population with advanced TR disease.

\section{TTVI: Procedural data, safety, and} acute procedural success

Overall, TR clip procedure was safe without any major procedural complications such as emergent surgery, cardiac tamponade, prolonged cardiopulmonary resuscitation, or myocardial infarction. The operator learning curve for TTVI has to be judged as steep. The procedure time of the first two TTVIs was 290 and $314 \mathrm{~min}$, which is rather long. However, the subsequent procedure time shortened and ranged between 54 and $200 \mathrm{~min}$ with a mean of $138.2 \pm 42.9 \mathrm{~min}$ (Table 2 ).

In total, $15(75 \%)$ patients had a successful intervention. In 5 (25\%) patients TTVI failed due to either unsuccessful clip implantation $(n=2)$, single leaflet device attachment (SLDA) before discharge $(\mathrm{n}=1)$, or TR reduction $<1$ grade $(\mathrm{n}=1)$. One patient developed upper gastrointestinal bleeding due to an esophageal Mallory-Weiss tear that needed endoscopic clip application after TTVI. However, there was no transfusion required. There was 1 death due to progressive cardiogenic shock 4 days after the procedure, but the procedure was planned as the last therapeutic option in this terminally ill patient ( $\mathrm{n}=1,5 \%$ in-hospital mortality).

A mean of $1.8 \pm 0.8$ clips were implanted per patient: in $14(70 \%)$ patients clips were implanted into the anteroseptal commissure, in 2 (10\%) patients solely in the posteroseptal commissure, whereas $3(15 \%)$ patients received clipping of both antero- and posteroseptal commissures (Table 2, Fig. 2). TR grade was significantly reduced from stage $4.0 \pm 0.8$ at baseline to stage $2.5 \pm 1.0$ at discharge and $2.8 \pm 0.8$ at 30 -day follow-up in all patients and particularly in patients with successful TTVI (Fig. 1A, B).

We did not see an association between clip location and success rate. However, the main jet location in patients with failed acute success was posteroseptal ( $\mathrm{n}=3,60 \%$; Table 2$)$. Of note, in 18 of 20 patients NTR MitraClips were implanted, and following the market launch of the new XTR clips the last 2 patients were successfully treated with XTR MitraClips.

Patients showed similar comorbidities irrespective of procedural success (Table 1). However, patients with a failed TTVI seemed to be sicker because the LVEF was lower, diuretics equivalent doses were higher, renal function was worse, and 
Table 1. Clinical characteristics of the patient population $(n=20)$ at baseline.

\begin{tabular}{|c|c|c|c|c|}
\hline & $\begin{array}{l}\text { All patients } \\
(n=20)\end{array}$ & $\begin{array}{l}\text { Acute success } \\
\qquad(\mathrm{n}=15)\end{array}$ & $\begin{array}{l}\text { Acute failure } \\
\quad(n=5)\end{array}$ & $\mathbf{P}$ \\
\hline \multicolumn{5}{|l|}{ Clinical characteristics } \\
\hline Age [years] & $78.6 \pm 8.3$ & $80.3 \pm 5.2$ & $73.4 \pm 13.8$ & 0.11 \\
\hline Male sex & $10(50 \%)$ & $6(39.9 \%)$ & $4(80 \%)$ & 0.13 \\
\hline Body mass index $\left[\mathrm{kg} / \mathrm{m}^{2}\right]$ & $27.2 \pm 5.7(27.2)$ & $27.1 \pm 6.2(27)$ & $27.2 \pm 4.5(27.4)$ & 0.97 \\
\hline NYHA class: & & & & 0.015 \\
\hline II & $2(10 \%)$ & $0(0 \%)$ & $2(40 \%)$ & \\
\hline III & $14(70 \%)$ & $11(73.3 \%)$ & $3(60 \%)$ & \\
\hline IV & $4(20 \%)$ & $4(26.7 \%)$ & $0(0 \%)$ & \\
\hline Ejection fraction $>40 \%$ & $16(80 \%)$ & $13(86.7 \%)$ & $3(60 \%)$ & 0.22 \\
\hline Edema & $18(90 \%)$ & $13(86.7 \%)$ & $5(100 \%)$ & 0.42 \\
\hline Pleural effusion & $10(50 \%)$ & $7(46.6 \%)$ & $3(60 \%)$ & 0.61 \\
\hline Ascites & $8(40 \%)$ & $4(26.7 \%)$ & $4(80 \%)$ & 0.036 \\
\hline STS score & $8.8 \pm 4.3(7.6)$ & $8.4 \pm 3.4(7.9)$ & $9.7 \pm 6.9(6.4)$ & 0.58 \\
\hline EuroScore II & $9.1 \pm 7.7(6.6)$ & $9.3 \pm 7.3(7.4)$ & $8.6 \pm 9.8(3.2)$ & 0.87 \\
\hline Concomitant mitral clip & $2(10 \%)$ & $2(13.3 \%)$ & $0(0 \%)$ & 0.42 \\
\hline \multicolumn{5}{|l|}{ Comorbidities } \\
\hline Atrial fibrillation & $19(95 \%)$ & $15(100 \%)$ & $4(80 \%)$ & 0.08 \\
\hline Diabetes & $6(30 \%)$ & $5(33.3 \%)$ & $1(20 \%)$ & 0.59 \\
\hline COPD & $3(15 \%)$ & $3(19.9 \%)$ & $0(0 \%)$ & 0.30 \\
\hline Pacemaker/ICD & $3(15 \%)$ & $2(13.3 \%)$ & $1(20 \%)$ & 0.74 \\
\hline Previous cardiac surgery & $3(15 \%)$ & $2(13.3 \%)$ & $1(20 \%)$ & 0.74 \\
\hline Pulmonary hypertension & $18(90 \%)$ & $14(93.3 \%)$ & $4(80 \%)$ & 0.42 \\
\hline $\begin{array}{l}\text { End stage renal failure } \\
\text { requiring dialysis }\end{array}$ & $2(10 \%)$ & $1(6.7 \%)$ & $1(20 \%)$ & 0.42 \\
\hline Coronary artery disease & $5(25 \%)$ & $2(13.3 \%)$ & $3(60 \%)$ & 0.34 \\
\hline \multicolumn{5}{|l|}{ Co-medication } \\
\hline $\begin{array}{l}\text { Torasemide equivalent } \\
\text { dose }[\mathrm{mg}]\end{array}$ & $24.2 \pm 15.6(20)$ & $20.7 \pm 14.6(20)$ & $37.5 \pm 12.6(40)$ & 0.052 \\
\hline Thiazide use & $9(45 \%)$ & $6(39.9 \%)$ & $3(60 \%)$ & 0.24 \\
\hline MRA & $4(20 \%)$ & $4(26.7 \%)$ & $0(0 \%)$ & 0.27 \\
\hline Oral anticoagulation & $15(75 \%)$ & $13(86.7 \%)$ & $3(60 \%)$ & 0.21 \\
\hline \multicolumn{5}{|l|}{ Laboratory parameters } \\
\hline Creatinine $[\mathrm{mg} / \mathrm{dL}]^{*}$ & $1.4 \pm 0.4(1.3)$ & $1.34 \pm 0.4(1.28)$ & $1.53 \pm 0.6(1.5)$ & 0.46 \\
\hline BUN $[\mathrm{mg} / \mathrm{dL}]^{*}$ & $77.8 \pm 46.1(71.9)$ & $73.1 \pm 37.1(71.9)$ & $93.4 \pm 73.1(90.1)$ & 0.46 \\
\hline $\mathrm{GFR}[\mathrm{mL} / \mathrm{min}]^{*}$ & $45.2 \pm 13.6(43.1)$ & $44.1 \pm 12.0(43.1)$ & $49.2 \pm 20.1(50.3)$ & 0.53 \\
\hline BNP $[p g / m L]$ & $826.7 \pm 734.8(540)$ & $806.9 \pm 657.2(622)$ & $886.2 \pm 1023.2(456)$ & 0.84 \\
\hline Bilirubin $[\mu \mathrm{mol} / \mathrm{L}]$ & $15.3 \pm 5.2(15)$ & $15.7 \pm 5.2(15)$ & $14.4 \pm 5.6(14)$ & 0.65 \\
\hline ASAT $[\mu \mathrm{mol} / L \times \mathrm{s}]$ & $0.52 \pm 0.22(0.46)$ & $0.54 \pm 0.25(0.48)$ & $0.44 \pm 0.13(0.45)$ & 0.42 \\
\hline ALAT $[\mu \mathrm{mol} / \mathrm{L} \times \mathrm{s}]$ & $0.29 \pm 0.2(0.22)$ & $0.31 \pm 0.21(0.28)$ & $0.21 \pm 0.12(0.17)$ & 0.36 \\
\hline Cholinesterasis $[\mu \mathrm{mol} / \mathrm{L} \times \mathrm{s}]$ & $98.1 \pm 31.2(99)$ & $102 \pm 32.7(103)$ & $86.2 \pm 25.5(78)$ & 0.35 \\
\hline Hemoglobin [mmol/L] & $7.6 \pm 0.8(7.8)$ & $7.5 \pm 0.9(7.6)$ & $7.8 \pm 0.4(7.9)$ & 0.24 \\
\hline
\end{tabular}

Data are shown as mean \pm standard deviation (median) or number (percentage). *Patients on dialysis were excluded from analysis of renal function parameters; NYHA - New York Heart Association; STS - Society of Thoracic Surgeons; COPD - chronic obstructive pulmonary disease; ICD — implantable cardioverter-defibrillator; MRA — mineralocorticoid receptor antagonists; BUN — blood urea nitrogen;

GFR - glomerular filtration rate; BNP — B-type natriuretic peptide; ASAT — aspartate transaminase; ALAT — alanine aminotransferase 
Table 2. Echocardiographic, invasive, and procedural characteristics of the patient population $(n=20)$.

\begin{tabular}{|c|c|c|c|c|}
\hline & $\begin{array}{l}\text { All patients } \\
(n=20)\end{array}$ & $\begin{array}{l}\text { Acute success } \\
\qquad(n=15)\end{array}$ & $\begin{array}{l}\text { Acute failure } \\
\quad(n=5)\end{array}$ & $\mathbf{P}$ \\
\hline \multicolumn{5}{|l|}{ Echocardiography } \\
\hline LVEF [\%] & $54.3 \pm 14.6(60.0)$ & $56.8 \pm 13.7(60)$ & $47.0 \pm 16.7(46)$ & 0.203 \\
\hline RV FAC [\%] & $35.0 \pm 6.3(33.5)$ & $35.1 \pm 6.6(33)$ & $34.8 \pm 6.1(37.5)$ & 0.95 \\
\hline TV annulus [mm] & $45.5 \pm 5.6(45.0)$ & $43.9 \pm 4.6(44)$ & $50.0 \pm 6.5(50)$ & 0.032 \\
\hline Coaptation depth [mm] & $7.4 \pm 3.2(7.0)$ & $6.7 \pm 3.1(6.8)$ & $9.8 \pm 2.6(11)$ & 0.056 \\
\hline Tenting area $\left[\mathrm{cm}^{2}\right]$ & $2.4 \pm 1.0(2.3)$ & $2.1 \pm 0.9(2.14)$ & $3.2 \pm 1.0(3.35)$ & 0.025 \\
\hline TR vena contracta $[\mathrm{mm}]$ & $11.5 \pm 4.3(10.0)$ & $11.5 \pm 4.2(10)$ & $12.6 \pm 4.6(12)$ & 0.53 \\
\hline EROA $\left[\mathrm{cm}^{2}\right]$ & $0.73 \pm 0.26(0.76)$ & $0.71 \pm 0.28(0.76)$ & $0.78 \pm 0.19(0.80)$ & 0.59 \\
\hline TR volume $[\mathrm{mL} / \mathrm{beat}]$ & $59.7 \pm 19.5(59)$ & $58.6 \pm 20.9(59)$ & $62.8 \pm 15.9(61)$ & 0.68 \\
\hline TR grade at baseline ( 5 stages): & $4.0 \pm 0.8(4)$ & $3.9 \pm 0.8(4)$ & $4.2 \pm 0.8(4)$ & 0.45 \\
\hline Severe & $7(35 \%)$ & $6(40 \%)$ & $1(20 \%)$ & 0.45 \\
\hline Massive & $7(35 \%)$ & $5(33.3 \%)$ & $2(40 \%)$ & \\
\hline Torrential & $6(30 \%)$ & $4(26.7 \%)$ & $2(40 \%)$ & \\
\hline Main jet location: & & & & 0.026 \\
\hline Central & $11(55 \%)$ & $9(60 \%)$ & $2(40 \%)$ & \\
\hline Anteroseptal & $5(25 \%)$ & $5(33.3 \%)$ & $0(0 \%)$ & \\
\hline Anteroposterior & $0(0 \%)$ & $0(0 \%)$ & $0(0 \%)$ & \\
\hline Posteroseptal & $4(20 \%)$ & $1(6.7 \%)$ & $3(60 \%)$ & \\
\hline \multicolumn{5}{|l|}{ Invasive hemodynamics } \\
\hline Systolic PP [mmHg] & $49.2 \pm 12.8(50.5)$ & $50.0 \pm 12.3(53)$ & $46.8 \pm 15.8(45)$ & 0.64 \\
\hline Mean PP [mmHg] & $31.3 \pm 7.5(31.0)$ & $31.3 \pm 6.8(32)$ & $31.2 \pm 10.2(30)$ & 0.99 \\
\hline Right atrial pressure $[\mathrm{mmHg}]$ & $12.9 \pm 5.6(14.0)$ & $12.6 \pm 5.7(12)$ & $13.6 \pm 5.6(15)$ & 0.74 \\
\hline PCWP [mmHg] & $20.6 \pm 5.6(20.0)$ & $20.9 \pm 5.3(20)$ & $19.6 \pm 7.1(17)$ & 0.68 \\
\hline $\mathrm{Cl}\left[\mathrm{L} / \mathrm{min} / \mathrm{m}^{2}\right]$ & $2.2 \pm 0.5(2.3)$ & $2.2 \pm 0.5(2.2)$ & $2.2 \pm 0.3(2.4)$ & 0.92 \\
\hline \multicolumn{5}{|l|}{ Tricuspid valve intervention } \\
\hline Procedure duration [min] & $170.0 \pm 75.8(150.5)$ & $163.6 \pm 65.6(140)$ & $127.4 \pm 60.9(114)$ & 0.29 \\
\hline Fluoroscopy time [min] & $18.8 \pm 15.3(15.4)$ & $19.5 \pm 13.3(16.2)$ & $17.1 \pm 11.9(12.1)$ & 0.73 \\
\hline Total number of clips & $1.8 \pm 0.8(2)$ & $1.7 \pm 0.6(2)$ & $1.4 \pm 1.1(1)$ & 0.50 \\
\hline Clip position: & & & & 0.23 \\
\hline Anteroseptal & $14(70 \%)$ & $10(66.7 \%)$ & $4(20 \%)$ & \\
\hline Posteroseptal & $2(10 \%)$ & $2(13.3 \%)$ & $0(0 \%)$ & \\
\hline Both & $3(15 \%)$ & $3(20 \%)$ & $0(0 \%)$ & \\
\hline Bleeding requiring transfusion & $2(10 \%)$ & $1(6.7 \%)$ & $1(20 \%)$ & 0.20 \\
\hline Acute renal failure & $3(15 \%)$ & $2(13.3 \%)$ & $1(20 \%)$ & 0.73 \\
\hline New dialysis & $1(5 \%)$ & $0(0 \%)$ & $1(20 \%)$ & 0.08 \\
\hline
\end{tabular}

Data are shown as mean \pm standard deviation (median) or number (percentage). LVEF - left ventricular ejection fraction; RV FAC - right ventricle fractional area change; TV — tricuspid valve; TR - tricuspid regurgitation; EROA - effective regurgitation orifice area; PP - pulmonary pressure; PCWP - pulmonary capillary wedge pressure; $\mathrm{Cl}$ - cardiac index

more often there was evidence of ascites, peripheral edema, and pleural effusion, even though not all parameters reached significance in this relatively small patient group (Table 1). Conversely, patients with failed and successful TTVI had comparable scores on STS and EuroScore II, but most of the aforementioned parameters are not included in these standardized scores, and neither are those validated for single TV procedures. Interestingly, we found no differences regarding invasive hemo- 


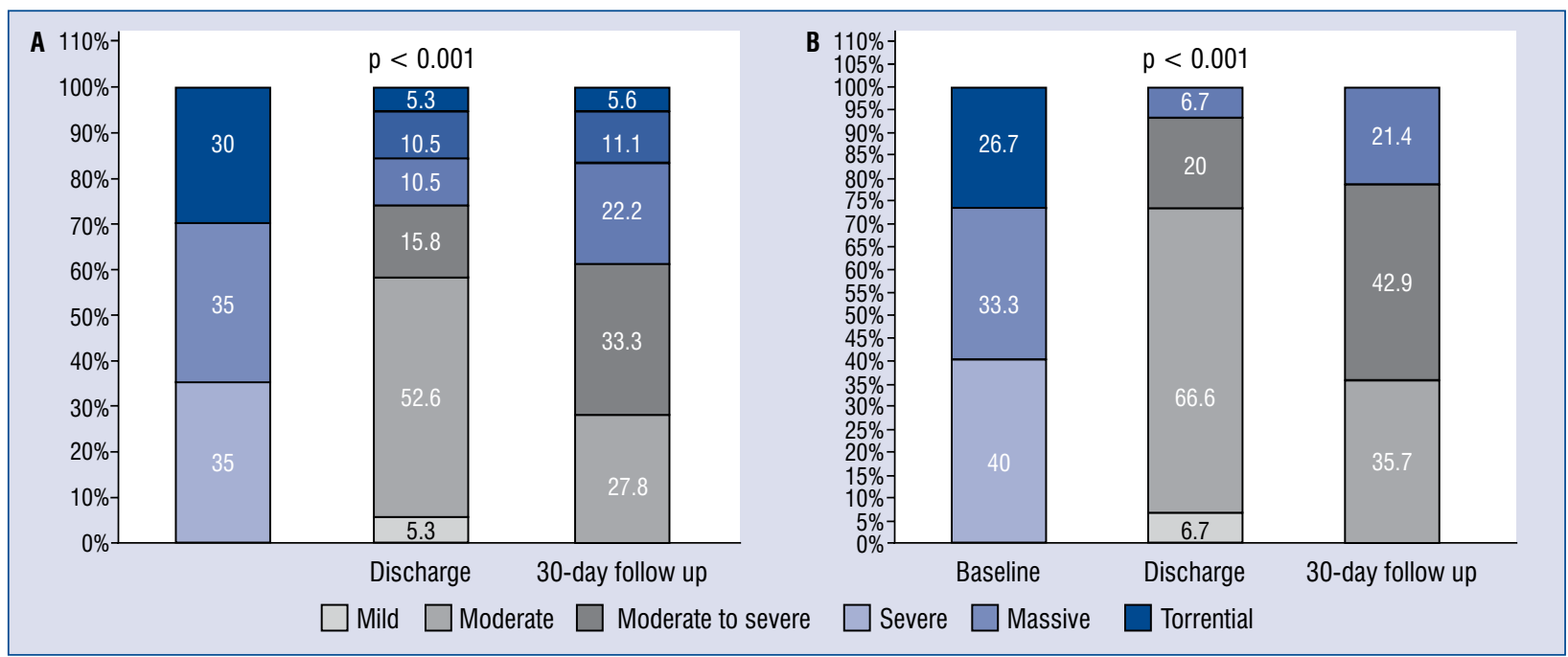

Figure 1. A. Tricuspid regurgitation grade pre- and post-tricuspid valve intervention in the total patient population $(n=20)$; One patient died before discharge and another patient died within 30 days; $\mathbf{B}$. Tricuspid regurgitation grade pre- and post-tricuspid valve intervention in patients with successful transcatheter tricuspid valve intervention at baseline $(n=15)$; One patient died before discharge and another patient died within 30 days.

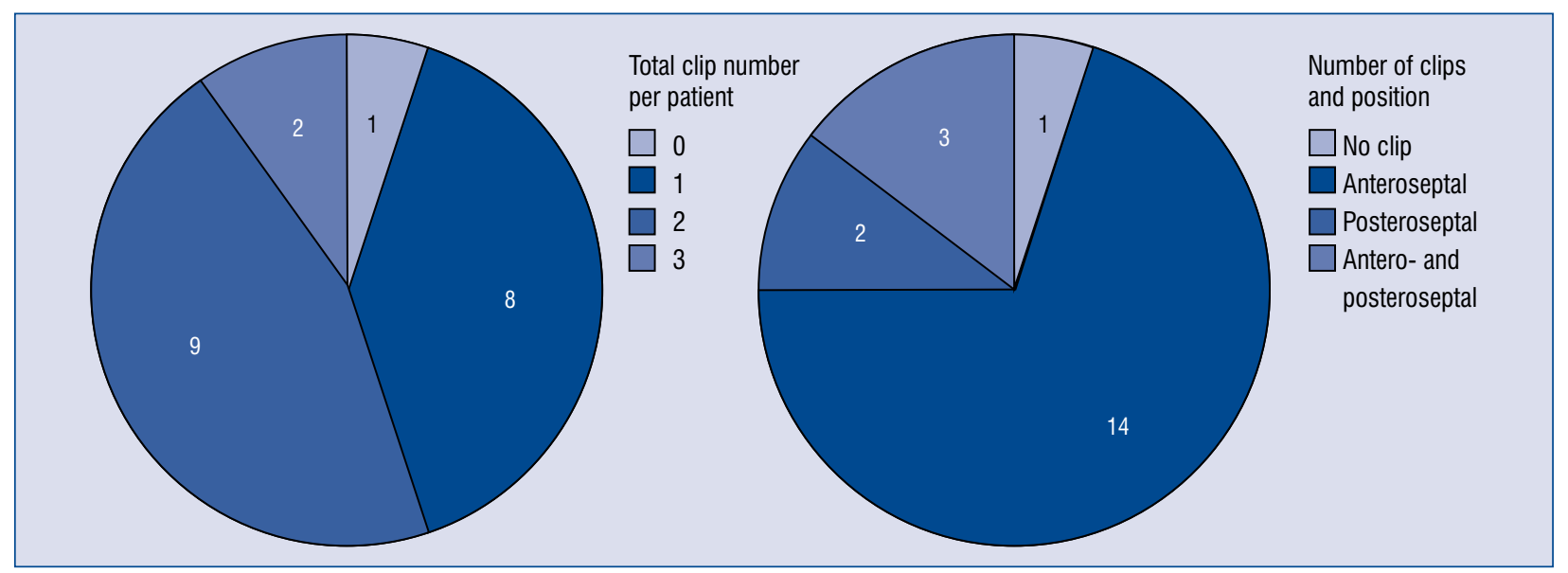

Figure 2. Numbers of clips implanted per patient (left) and leaflet location of implanted clips (right).

dynamics and procedural aspects, as well as RV function (Table 2). There were no differences in conventional echocardiographic parameters for TR severity (vena contracta, EROA, TR grade, TR volume; Table 2). However, echocardiographic parameters describing tricuspid annulus geometry (TV annulus, coaptation depth, tenting area) were significantly larger in patients with failed TTVI (Table 2).

\section{Clinical outcome at 30-day follow-up}

The NYHA class improved significantly between baseline and 30-day follow-up, as outlined in Table 3 and Figure 3. At 30-day follow-up, SLDA with worsening of TR was observed in 2 more patients, and 1 patient died due to worsening HF. Thus, the overall 30-day overall success rate was $60 \%(\mathrm{n}=12)$ and 30 -day mortality was $10 \%(\mathrm{n}=2)$. However, the deaths in this cohort were due to progressive and terminal HF despite TTVI rather than due to TTVI. Comparing patients at 30-day follow-up (30-day success, $\mathrm{n}=12$ vs. 30-day failure, $n=8$ ), we still observed no differences in conventional echocardiographic parameters as mentioned above. In line with the reported acute success data, tricuspid annulus ge- 
Table 3. Outcome parameters at discharge and 30-day follow-up in the overall patient population and comparing patients with acute clinical and procedural success versus acute failure.

\begin{tabular}{lcccc}
\hline & $\begin{array}{c}\text { All patients } \\
(\mathbf{n}=\mathbf{2 0})\end{array}$ & $\begin{array}{c}\text { Acute success } \\
(\mathbf{n}=\mathbf{1 5})\end{array}$ & $\begin{array}{c}\text { Acute failure } \\
(\mathbf{n}=\mathbf{5})\end{array}$ & $\mathbf{P}$ \\
\hline TR grade at discharge & $\mathbf{2 . 5} \pm \mathbf{0 . 9 6}(\mathbf{2 . 0 )}$ & $\mathbf{2 . 1} \pm \mathbf{0 . 5}(\mathbf{2 . 0 )}$ & $\mathbf{4 . 0} \pm \mathbf{0 . 8}(\mathbf{4 . 0 )}$ & $<\mathbf{0 . 0 0 1}$ \\
TR grade at 30-day follow-up & $\mathbf{2 . 8} \pm \mathbf{0 . 8 ( 2 . 5 )}$ & $\mathbf{2 . 4} \pm \mathbf{0 . 4 ( 2 . 5 )}$ & $\mathbf{4 . 0} \pm \mathbf{0 . 8}(\mathbf{4 . 0 )}$ & $<\mathbf{0 . 0 0 1}$ \\
In-hospital mortality & $1(5 \%)$ & $0(0 \%)$ & $1(20 \%)$ & 0.99 \\
30-day mortality & $2(10 \%)$ & $1(6.7 \%)$ & $1(20 \%)$ & 0.42 \\
Total hospital stay [days] & $12.4 \pm 7.5(8.5)$ & $11.5 \pm 6.5(9)$ & $15.0 \pm 10.1(8)$ & 0.37 \\
ICU stay [days] & $3.6 \pm 3.9(2.5)$ & $3.6 \pm 4.5(2)$ & $3.4 \pm 1.8(4)$ & 0.90 \\
SLDA & $3(15 \%)$ & $2(13.3 \%)$ & $1(20 \%)$ & N/A \\
Time to SLDA [days] & $26 \pm 19.1(37)$ & $37.0 \pm 0.0(37)$ & 4 & N/A \\
\hline
\end{tabular}

Data are shown as mean \pm standard deviation (median) or number (percentage); TR — tricuspid regurgitation; ICU — intensive care unit; SLDA - single leaflet device attachment

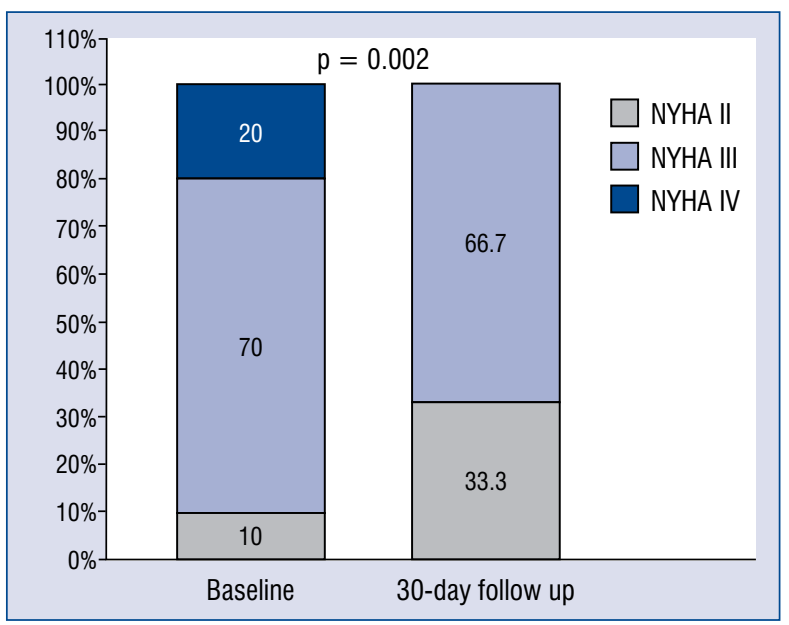

Figure 3. New York Heart Association (NYHA) class at baseline and 30-day follow-up in the total patient population ( $\mathrm{n}=20$ ); Two patients died within 30 days.

ometry parameters remained larger in the 30 -day failure group (tenting area $2.0 \pm 0.8$ vs. $2.9 \pm 1.1 \mathrm{~cm}^{2}$, $\mathrm{p}=0.057$; coaptation depth $6.1 \pm 2.4$ vs. $9.5 \pm$ $\pm 3.3, \mathrm{p}=0.016$; TV annulus $44.6 \pm 4.5$ vs. $46.8 \pm$ $\pm 7.1, \mathrm{p}=0.412)$. Of note, in all patients $(\mathrm{n}=3)$ with a transvalvular implanted lead (cardiac implantable electronic devices [CIED]) TTVI failed at 30-day follow-up $(\mathrm{p}=0.02)$.

\section{Discussion}

In this prospective cohort study, we analyzed clinical, procedural, and echocardiographic data on outcomes after TTVI using the edge-to-edge technique. With high initial implantation success
(85\%) and low in-hospital mortality rate (5\%), this procedure is a promising alternative to surgical TV repair or replacement. While our observed 30-day success rate was $60 \%$ lower than that reported by other investigators (e.g. 86\% in TRILUMINATE and TriValve, $81 \%$ by Besler et al. [24]), patient selection seems to play a pivotal role because we included patients with advanced (right-sided) HF $[7,24-26]$. The high TRuE risk score of $>6$ in our cohort underlines this disease stage and a patient population at high risk [23].

The optimal timing for surgical or transcatheter TV repair remains unclear. RV and tricuspid annular remodeling progress with advanced TR stages and RV failure. Therefore, early TV intervention during TR disease progression using the edge-to-edge technique has been suggested to reverse RV remodeling and failure. Echocardiographic grading of TR severity is complicated and requires extensive operator training due to the complexity of the TV apparatus and its challenging anatomy. Interestingly, we found no differences in "conventional" echocardiographic parameters for TR severity (vena contracta, EROA, TR grade, TR volume) with respect to short-term success. Recently, more sophisticated approaches involving qualitative, quantitative, and semi-quantitative criteria have been proposed. These data require further verification regarding their predictive power [7].

Moreover, identification of the main pathogenic mechanism for TR might have prognostic implications [7]. A decade ago, Min et al. [27] demonstrated that the antero-posterior annulus diameter and tenting volume before tricuspid annuloplasty were independent predictors of residual 
TR after surgical correction. Our data underline this observation. We show differences in tricuspid annulus geometry expressed as TV annulus diameter, coaptation depth, and tenting area, which were all significantly larger in the patients with failed percutaneous edge-to-edge repair of TV. However geometric changes of the TV apparatus seem to be an indicator of severity of TR. Therefore, coaptation enhancement strategies like the edge-to-edge technique might be a feasible approach during mid-stage TR disease. Conversely, advanced TR disease is characterized by progressive annular dilatation, RV remodeling, and leaflet tethering, which then result in geometric abnormalities of the TV apparatus. In light of this, combined approaches with additional annuloplasty or TV replacement strategies might be the preferable treatment option for later stages of TR. On the other hand, there might be "a point of no return" during TR disease progression, reflecting the underlying left and/or right $\mathrm{HF}$. As we know from the published trials COAPT and MITRA-FR for percutaneous treatment of functional mitral regurgitation, valve regurgitation needs also to be graded and judged with respect to atrial and ventricular dimensions and function [28, 29].

In our patient cohort, TTVI failed in most patients with the main jet located non-centrally and non-anteroseptally (success rate $25 \%, 1$ out of 4 ). However, jet location can be verified as a predictor for procedural outcome of the edge-to-edge repair and is in agreement with previously published data $[22,24]$. Only 3 of our patients $(15 \%)$ had a CIED, compared to $26 \%$ in the TriValve registry. However, all 3 CIED patients failed TTVI at 30-day follow-up, which is contrary to the reported success rate of $78.6 \%$ in the TriValve cohort [30]. This difference in outcome might be related to our sample size or possibly due to patient selection. The main jet location in the TriValve registry was in the favorable central/ /anteroseptal location in CIED patients, which is contrary to our CIED patients [30].

Comparing our patient data with those of a previously published large cohort study on FTR, there are distinct differences regarding the patient population [13]. Contrary to Benfari et al. [13], our cohort is of mixed nature regarding LV function including both $\mathrm{HFrEF}$ and $\mathrm{HF}$ with preserved ejection fraction patients. Moreover, we found atrial fibrillation in all of our patients compared to only $48 \%$ of the severe FTR patients in the Benfari cohort. Atrial fibrillation is seen as a major contributor to FTR and is strongly related to severe FTR because it can lead to right atrium and thus annulus dilation even in the absence of pulmonary hypertension [31, 32]. However, because FTR is related to a variety of cardiac dysfunction, detailed classification of associated TR pathology, especially for the group of functional TR, and studies on therapeutic options with respect to the origin of TR disease are needed. On the other hand, studies on surgical TR repair could show that survival is not affected by the cause of TR but rather by HF stage and comorbidities [18].

Some studies reported excess mortality for FTR as a result of underlying pulmonary hypertension and/or right/left HF [33-35]. This concept has been challenged in the past years, because several studies could show that FTR is an independent predictor for survival $[13,16,17]$. This calls not only for a specific treatment of TR but also for an effort on early identification of FTR patients; in particular, if other cardiac pathologies such as atrial fibrillation are present with the resulting need for close clinical monitoring.

\section{Limitations of the study}

This is an observational, prospective cohort study reporting a single-center experience with a small sample size and multiple comparisons. Thus, patient bias and significances appearing by chance cannot be excluded completely. The findings of our study have to be judged as hypothesis-generating and should be verified by further studies. However, the overall available patient population for isolated TV procedures is limited. In other studies, such as the recently published Triluminate registry, an average of only 4 patients per study site was included during a comparable time period [25].

\section{Conclusions}

Our data suggest the significance of tricuspid annulus geometry, which seems to impact short-term outcomes after percutaneous edge-to-edge repair of TR. Further investigations and possibly more sophisticated interventional approaches involving the tricuspid annulus are needed to better understand and treat this complex valve disease.

\section{Conflict of interest: None declared}

\section{References}

1. Baumgartner H, Falk V, Bax JJ, et al. 2017 ESC/EACTS Guidelines for the management of valvular heart disease. Eur Heart J. 2017; 38(36): 2739-2791, doi: 10.1093/eurheartj/ehx391, indexed in Pubmed: 28886619. 
2. Chikwe J, Itagaki S, Anyanwu A, et al. Impact of concomitant tricuspid annuloplasty on tricuspid regurgitation, right ventricular function, and pulmonary artery hypertension after repair of mitral valve prolapse. J Am Coll Cardiol. 2015; 65(18): 1931-1938, doi: 10.1016/j.jacc.2015.01.059, indexed in Pubmed: 25936265.

3. Zack CJ, Fender EA, Chandrashekar P, et al. National trends and outcomes in isolated tricuspid valve surgery. J Am Coll Cardiol. 2017; 70(24): 2953-2960, doi: 10.1016/j.jacc.2017.10.039, indexed in Pubmed: 29241483.

4. Navia JL, Brozzi NA, Klein AL, et al. Moderate tricuspid regurgitation with left-sided degenerative heart valve disease: to repair or not to repair? Ann Thorac Surg. 2012; 93(1): 59-67; discussion 68, doi: 10.1016/j.athoracsur.2011.08.037, indexed in Pubmed: 22093694.

5. Benedetto U, Melina G, Angeloni E, et al. Prophylactic tricuspid annuloplasty in patients with dilated tricuspid annulus undergoing mitral valve surgery. J Thorac Cardiovasc Surg. 2012; 143(3): 632-638, doi: 10.1016/j.jtcvs.2011.12.006, indexed in Pubmed: 22244561.

6. Kim JB, Yoo DG, Kim GS, et al. Mild-to-moderate functional tricuspid regurgitation in patients undergoing valve replacement for rheumatic mitral disease: the influence of tricuspid valve repair on clinical and echocardiographic outcomes. Heart. 2012; 98(1): 24-30, doi: 10.1136/heartjnl-2011-300403, indexed in Pubmed: 21930721.

7. Taramasso M, Benfari G, van der Bijl P, et al. Transcatheter versus medical treatment of patients with symptomatic severe tricuspid regurgitation. J Am Coll Cardiol. 2019; 74(24): 2998-3008, doi: 10.1016/j.jacc.2019.09.028, indexed in Pubmed: 31568868.

8. Addetia K, Muraru D, Veronesi F, et al. 3-Dimensional echocardiographic analysis of the tricuspid annulus provides new insights into tricuspid valve geometry and dynamics. JACC Cardiovasc Imaging. 2019; 12(3): 401-412, doi: 10.1016/j.jcmg.2017.08.022, indexed in Pubmed: 29153573.

9. Chang JD, Manning WJ, Ebrille E, et al. Tricuspid valve dysfunction following pacemaker or cardioverter-defibrillator implantation. J Am Coll Cardiol. 2017; 69(18): 2331-2341, doi: 10.1016/j. jacc.2017.02.055, indexed in Pubmed: 28473139.

10. Hahn RT, Zamorano JL. The need for a new tricuspid regurgitation grading scheme. Eur Heart J Cardiovasc Imaging. 2017; 18(12): 1342-1343, doi: 10.1093/ehjci/jex139, indexed in Pubmed: 28977455.

11. Badano LP, Hahn R, Rodríguez-Zanella H, et al. Morphological assessment of the tricuspid apparatus and grading regurgitation severity in patients with functional tricuspid regurgitation: thinking outside the box. JACC Cardiovasc Imaging. 2019; 12(4): 652-664, doi: 10.1016/j.jcmg.2018.09.029, indexed in Pubmed: 30947907.

12. Kilic A, Saha-Chaudhuri P, Rankin JS, et al. Trends and outcomes of tricuspid valve surgery in North America: an analysis of more than 50,000 patients from the Society of Thoracic Surgeons database. Ann Thorac Surg. 2013; 96(5): 1546-52; discussion 1552, doi: 10.1016/j.athoracsur.2013.06.031, indexed in Pubmed: 24070702 .

13. Benfari G, Antoine C, Miller WL, et al. Excess mortality associated with functional tricuspid regurgitation complicating heart failure with reduced ejection fraction. Circulation. 2019; 140(3): 196-206, doi: 10.1161/CIRCULATIONAHA.118.038946, indexed in Pubmed: 31117814.

14. Topilsky Y, Maltais S, Medina Inojosa J, et al. Burden of tricuspid regurgitation in Patients diagnosed in the community setting.
JACC Cardiovasc Imaging. 2019; 12(3): 433-442, doi: 10.1016/j. jcmg.2018.06.014, indexed in Pubmed: 30121261.

15. Topilsky Y, Nkomo VT, Vatury O, et al. Clinical outcome of isolated tricuspid regurgitation. JACC Cardiovasc Imaging. 2014; 7(12): 1185-1194, doi: 10.1016/j.jcmg.2014.07.018, indexed in Pubmed: 25440592.

16. Nath J, Foster E, Heidenreich PA. Impact of tricuspid regurgitation on long-term survival. J Am Coll Cardiol. 2004; 43(3): 405-409, doi: 10.1016/j.jacc.2003.09.036, indexed in Pubmed: 15013122.

17. Agricola E, Marini C, Stella S, et al. Effects of functional tricuspid regurgitation on renal function and long-term prognosis in patients with heart failure. J Cardiovasc Med (Hagerstown). 2017; 18(2): 60-68, doi: 10.2459/JCM.0000000000000312, indexed in Pubmed: 26258726.

18. Kim JB, Jung SH, Choo SJ, et al. Surgical outcomes of severe tricuspid regurgitation: predictors of adverse clinical outcomes. Heart. 2013; 99(3): 181-187, doi: 10.1136/heartjnl-2012-302856, indexed in Pubmed: 23038792.

19. Ratnatunga C, Edwards MB, Dore C, et al. Tricuspid valve replacement: UK heart valve registry mid-term results comparing mechanical and biological prostheses. Ann Thoracic Sur. 1998; 66(6): 1940-1947, doi: 10.1016/s0003-4975(98)01183-7.

20. Moraca RJ, Moon MR, Lawton JS, et al. Outcomes of tricuspid valve repair and replacement: a propensity analysis. Ann Thorac Surg. 2009; 87(1): 83-88, doi: 10.1016/j.athoracsur.2008.10.003, indexed in Pubmed: 19101275.

21. Chang CC, Veen KM, Hahn RT, et al. Uncertainties and challenges in surgical and transcatheter tricuspid valve therapy: a stateof-the-art expert review. Eur Heart J. 2020; 41(20): 1932-1940, doi: 10.1093/eurheartj/ehz614, indexed in Pubmed: 31511897.

22. Mehr M, Taramasso M, Besler C, et al. 1-Year outcomes after edge-to-edge valve repair for symptomatic tricuspid regurgitation: results from the trivalve registry. JACC Cardiovasc Interv. 2019; 12(15): 1451-1461, doi: 10.1016/j.jcin.2019.04.019, indexed in Pubmed: 31395215.

23. Alushi B, Beckhoff F, Leistner DM, et al. 5938 Mortality risk stratification in patients with severe tricuspid regurgitation: Insights from the Tricuspid Regurgitation REgistry (TRuE). Eur Heart J. 2019; 40(Suppl 1), doi: 10.1093/eurheartj/ehz746.0088.

24. Besler C, Orban M, Rommel KP, et al. Predictors of procedural and clinical outcomes in patients with symptomatic tricuspid regurgitation undergoing transcatheter edge-to-edge repair. JACC Cardiovasc Interv. 2018; 11(12): 1119-1128, doi: 10.1016/j. jcin.2018.05.002, indexed in Pubmed: 29929631.

25. Nickenig G, Weber M, Schueler R, et al. 6-Month outcomes of tricuspid valve reconstruction for patients with severe tricuspid regurgitation. J Am Coll Cardiol. 2019; 73(15): 1905-1915, doi: 10.1016/j.jacc.2019.01.062, indexed in Pubmed: 30999993.

26. Braun D, Nabauer M, Orban M, et al. One-year results of transcatheter treatment of severe tricuspid regurgitation using the edge-to-edge repair technique. EuroIntervention. 2018; 14(4): e413-e415, doi: 10.4244/EIJ-D-18-00186, indexed in Pubmed: 29741485.

27. Min SY, Song JM, Kim JH, et al. Geometric changes after tricuspid annuloplasty and predictors of residual tricuspid regurgitation: a real-time three-dimensional echocardiography study. Eur Heart J. 2010; 31(23): 2871-2880, doi: 10.1093/eurheartj/ehq227, indexed in Pubmed: 20601392.

28. Stone GW, Lindenfeld J, Abraham WT, et al. Transcatheter mitral-valve repair in patients with heart failure. N Engl J Med. 
2018; 379(24): 2307-2318, doi: 10.1056/NEJMoa1806640, indexed in Pubmed: 30280640.

29. Obadia JF, Messika-Zeitoun D, Leurent G, et al. Percutaneous repair or medical treatment for secondary mitral regurgitation. N Engl J Med. 2018; 379(24): 2297-2306, doi: 10.1056/NEJMoa1805374, indexed in Pubmed: 30145927.

30. Taramasso M, Gavazzoni M, Pozzoli A, et al. Outcomes of TTVI in patients with pacemaker or defibrillator leads: data from the trivalve registry. JACC Cardiovasc Interv. 2020; 13(5): 554-564, doi: 10.1016/j.jcin.2019.10.058, indexed in Pubmed: 31954676.

31. Topilsky Y, Khanna A, Le Tourneau T, et al. Clinical context and mechanism of functional tricuspid regurgitation in patients with and without pulmonary hypertension. Circ Cardiovasc Imaging. 2012; 5(3): 314-323, doi: 10.1161/CIRCIMAGING.111.967919, indexed in Pubmed: 22447806.
32. Zhou X, Otsuji Y, Yoshifuku S, et al. Impact of atrial fibrillation on tricuspid and mitral annular dilatation and valvular regurgitation. Circ J. 2002; 66(10): 913-916, doi: 10.1253/circj.66.913, indexed in Pubmed: 12381084.

33. Mutlak D, Lessick J, Khalil S, et al. Tricuspid regurgitation in acute heart failure: is there any incremental risk? Eur Heart J Cardiovasc Imaging. 2018; 19(9): 993-1001, doi: 10.1093/ehjci/ jex343, indexed in Pubmed: 29346535.

34. Neuhold S, Huelsmann M, Pernicka E, et al. Impact of tricuspid regurgitation on survival in patients with chronic heart failure: unexpected findings of a long-term observational study. Eur Heart J. 2013; 34(11): 844-852, doi: 10.1093/eurheartj/ehs465, indexed in Pubmed: 23335604.

35. Koelling TM, Aaronson KD, Cody RJ, et al. Prognostic significance of mitral regurgitation and tricuspid regurgitation in patients with left ventricular systolic dysfunction. Am Heart J. 2002; 144(3): 524-529, doi: 10.1067/mhj.2002.123575, indexed in Pubmed: 12228791. 